\title{
Scientific/Technical report
}

DE-FG02-08ER64579

Nanoimaging to Prevent and Treat Alzheimer's and Parkinson's Diseases

Principal Investigator: Yuri Lyubchenko, University of Nebraska Medical Center

Collaborators:

Dr. Keana, University of Oregon

Dr. Robert Jernigan, Iowa State University

Dr. Chris Rochet, Purdue University

\section{Summary of the original project}

It is now known that Alzheimer's, Parkinson's and Huntington's diseases result from proteins piling up instead of forming their normal structure in the brain. This protein build up blocks the transmission of signals to, from and within the brain, causing loss of memory, tremors, and other clinical symptoms associated with neurological diseases. The prevalence of disease is increasing and without suitable treatments or cure will have staggering socioeconomic effects. Indeed, AD is now the sixth-leading cause of death and the only cause of death among the top 10 in the United States. Based on 2000-2008 mortality data from the National Center for Health Statistics, $\mathrm{AD}$ death rates have risen 66 percent. There are no survivors for $\mathrm{AD}$ and other diseases as well as no treatment. This project will develop innovative approaches to characterization of the very early stages of protein aggregation that eventually can be translated to the development of early diagnostic tools and efficient treatments for Alzheimer's, Parkinson's and Huntington's diseases. Funding will be used to acquire nanoimaging technology for nanoscale imaging, manipulation and analysis of biomedical materials to develop treatments that will repair disabled proteins and cure diseases that result from protein malfunction, specifically Alzheimer's and Parkinson's diseases. Expected outcomes include tests for early diagnosis and therapeutic treatments for these devastating neurological diseases.

\section{Goals of the project}

The first and perhaps most important elements in most neurodegenerative processes are misfolding and aggregation of specific proteins. Despite the crucial importance of protein misfolding and abnormal interactions, very little is currently known about the molecular mechanism underlying these processes. Factors that lead to protein misfolding and aggregation in vitro are poorly understood, in addition to the complexities involved in the formation of protein nanoparticles with different morphologies (e.g. nanopores and other species) in vivo. A clear understanding of the molecular mechanisms of misfolding and aggregation will facilitate rational approaches to prevent protein misfolding mediated pathologies. This knowledge, in conjunction with molecular modeling, will fundamentally advance our understanding of cellular nanomachines and their function during protein deposition disorders. This application focusing 
on protein misfolding and early stages of aggregation will advance both short and long-term goals at the basic and applied therapeutic levels.

The following three major aims are proposed:

Aim 1 To elucidate the mechanisms of protein misfolding, we will establish an extensive program of experimental studies using a broad arsenal of advanced nanoscale and traditional techniques that will be integrated with molecular-scale modeling of protein misfolding and the nucleation of aggregate structures.

Aim 2 To identify intracellular machinery or/and multicomponent complexes critically involved in protein misfolding, we will characterize interactions between targeted proteins and specific intracellular components or metabolites that impact on protein conformational pathways leading to protein misfolding accompanied by formation of toxic aggregated morphologies.

Aim 3 To design innovative nanotechnology tools for the control of intracellular protein misfolding and aggregation processes, we will develop a predictive molecular scale model for intracellular protein misfolding and the formation of toxic aggregates. Verified through experimental studies under aim " 1 ", the objective is to establish an enabling foundation for the engineering of novel molecular diagnostics and therapeutics for various cellular pathologies.

Accomplishing the goals of our first objective will provide a fundamental understanding of protein misfolding phenomena and lead to a predictive model for self-assembly of aggregates. Achieving the other two objectives will provide the molecular biological level basis for elucidating pathways of protein misfolding and aggregation within cells, identifying pathways or corresponding molecular mechanisms that will reveal opportunities for intervention through novel types of nanomedicine approaches. The strategy of this proposal is entirely different from traditional drug development approaches targeting protein aggregates without having methods available for early detection of misfolding. Protein misfolding is the first step in a long chain of events in development of protein misfolding diseases, so early detection of aberrant pathways and shutting down or repairing these pathologic pathways will allow us to prevent the disease and possibly cure it.

\section{Accomplishments}

We were able to accomplish all specific aims of the projects and the results of the studies for each specific aim are briefly summarized below.

\section{Specific aim 1. Molecular mechanism of protein misfolding}

a. Experimental studies. The single molecule AFM probing approach was applied to a number of proteins and peptide undergoing self-assembly into amyloid-type aggregates. We were able to characterize the misfolded states not only by their strength, but also to measure the lifetimes of the dimers [1]. A number of important conclusions emerge from these studies. One of them is the lifetime of misfolded dimeric complexes - this parameter is within the range of a second. It is instructive to discuss these numbers along the line of the protein dynamics schematically shown in Fig. 1. Each protein is a dynamic system capable of adopting various transient states, along with the most stable native state, termed here a "folded monomer". Transient states, including fully unfolded and misfolded states, are shown in the scheme. Depending on the scale of the dynamics, the timescale of local protein dynamics is in the range 
of nanoseconds and large-scale dynamics may occur in the microsecond time scale. Computational analysis based on Molecular Dynamics (MD) approach showed the range of 10ns $-1 \mu \mathrm{s}\left(10^{-9}-10^{-6} \mathrm{~s}\right)$ as a time scale for the protein dynamics that involve the formation of misfolded conformations. When two dynamic misfolded proteins interact to form a dimer, the system can live as long as a second. Compared to the dynamics of monomeric proteins, the misfolded conformation of the protein in the dimeric state is $10^{6}$ times more stable. This is a very important finding, suggesting that the formation of dimers leads to enormous stabilization of the protein misfolded state; in other words, dimerization is the mechanism by which a misfolded state of the protein is stabilized.

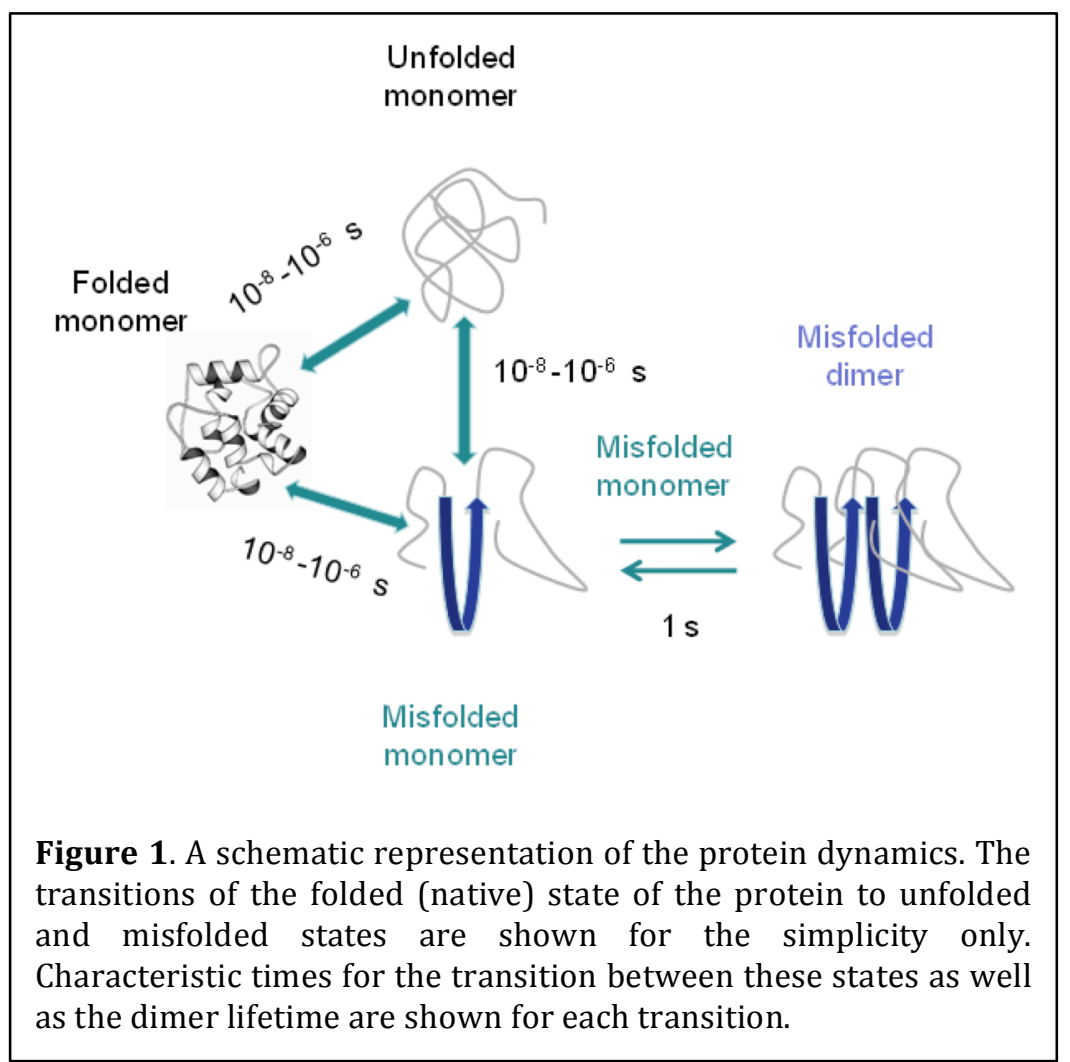

The high stability of dimers means that the probability of the aggregation steps with participation of dimers is $10^{6}$ times higher compared to a reaction without them. For example, the probability of tetramer formation with the assembly of two dimers will be $10^{6}$ times higher compared to the formation of trimers via the interaction of dimer and monomer. Note a recent publication in which $\mathrm{A} B$ peptide oligomers with only even numbers of monomers are formed [2]. High stability of the dimers provides the explanation for these findings. Additionally, the spectroscopic analysis of cross-linked dimers of $A ß$ peptides along with other oligomeric forms revealed a dramatic change of the peptide conformation within the dimers compared to the monomeric states [3]. There were also changes between other oligomers, but these were comparatively minor ones. Another recent publication [4] analyzed dimers of Aß peptides isolated from brain. It was found that dimers are abundant species isolated from the brain and they have a high neurotoxicity. Recent studies show that naturally secreted A 3 dimers and trimers induce progressive loss of hippocampal synapses [5, 6] It has been recently discussed that oxidation is able to stabilize the dimers in vitro as well as within the cell [7].

Altogether, the published data and our results confirm our hypothesis on a key role of dimers in the amyloidosis. According to our model, the formation of dimers is a mechanism by which a misfolded state of the protein is stabilized to a significant extent. Due to a long lifetime, the probability of further interactions involving dimers dramatically increases. Therefore, the ability to form long lifetime dimers is a fundamental property of the misfolded states of the protein, and this dimer formation triggers the protein aggregation process. The described model 
is a paradigm shift of the protein misfolding and aggregation phenomenon, pointing to a critical role of misfolded dimer formation in this process.

Importantly, the proposed model poses dimers as major targets in the development of the treatments for protein misfolding diseases. The search of efficient diagnostics should be focused on the detection of dimers, a large concentration of which will trigger a rapid aggregation process. The dimers should be targets in the development of efficient preventions, so finding the means for their dissociation would be a straightforward approach in this direction. AFM force spectroscopy is a very useful approach in the search of appropriate candidates, as the destabilization effect of a potential compound can be characterized quantitatively. Although the dimers are stable enough to shift the conformation equilibrium of the protein conformational dynamics to the aggregation pathway, higher oligomers such as trimers, tetramers and so on would be also important for the aggregate growth, and their property should be characterized in order to get a complete picture of the process. The DFS studies of various oligomers can help identify species (nuclei) that are responsible for the fast growth of fibrils. Conformational transitions within such nuclei are required for triggering the fibrillation step and these transitions are dependent on the oligomer size.

b. Computational analysis (collaboration with R. Jernigan, Iowa State University). This project has required us to develop several new tools for the investigation of peptide aggregation by computations:

1. We finished developing the database of known structural fragments that include multiple structures of the same sequence fragment, and we now have a web interface for a publicly available server that is ready to be deployed. We expect that this to be available very soon.

2. We have also been applying our database of fragments to improve predictions of protein secondary structures, predictions of intrinsically disordered regions in protein structures, predictions of phosphorylation and other post-transitional modification sites and predictions of parts of proteins that are likely to have alternative forms in proteins. The fragment database can be used for extracting information about both structural and sequence fragments that can undergo conformational transitions. This database is being applied to multiple problems, beyond our original intentions.

3. We have developed a database of knowledge-based coarse-grained potentials on the publicly available website server: http://gor.bb.iastate.edu/potential. Our new knowledgebased potential server makes these potentials available for easy use to automatically compute the energies of protein structures or models supplied by the user. Users can select potentials according to their needs and preferences.

4. We have recently employed molecular dynamics (MD) simulations to test our model on the protein misfolding suggesting that interprotein interaction stabilizes misfolded dimers.

For this study, we selected 13-23 segment of $A \beta$ peptide since it contains the region $A \beta(16-$ $20)$, which is crucial for $A \beta$ fibril formation and mediates the strongest $A \beta$ - $A \beta$ binding. The AFM experiments demonstrate that the peptide spontaneously forms amyloid fibrils and the 
peptide dimers are stable with the lifetime $\sim 1 \mathrm{~s}$. The results of the MD simulations are summarized in Fig. 2.

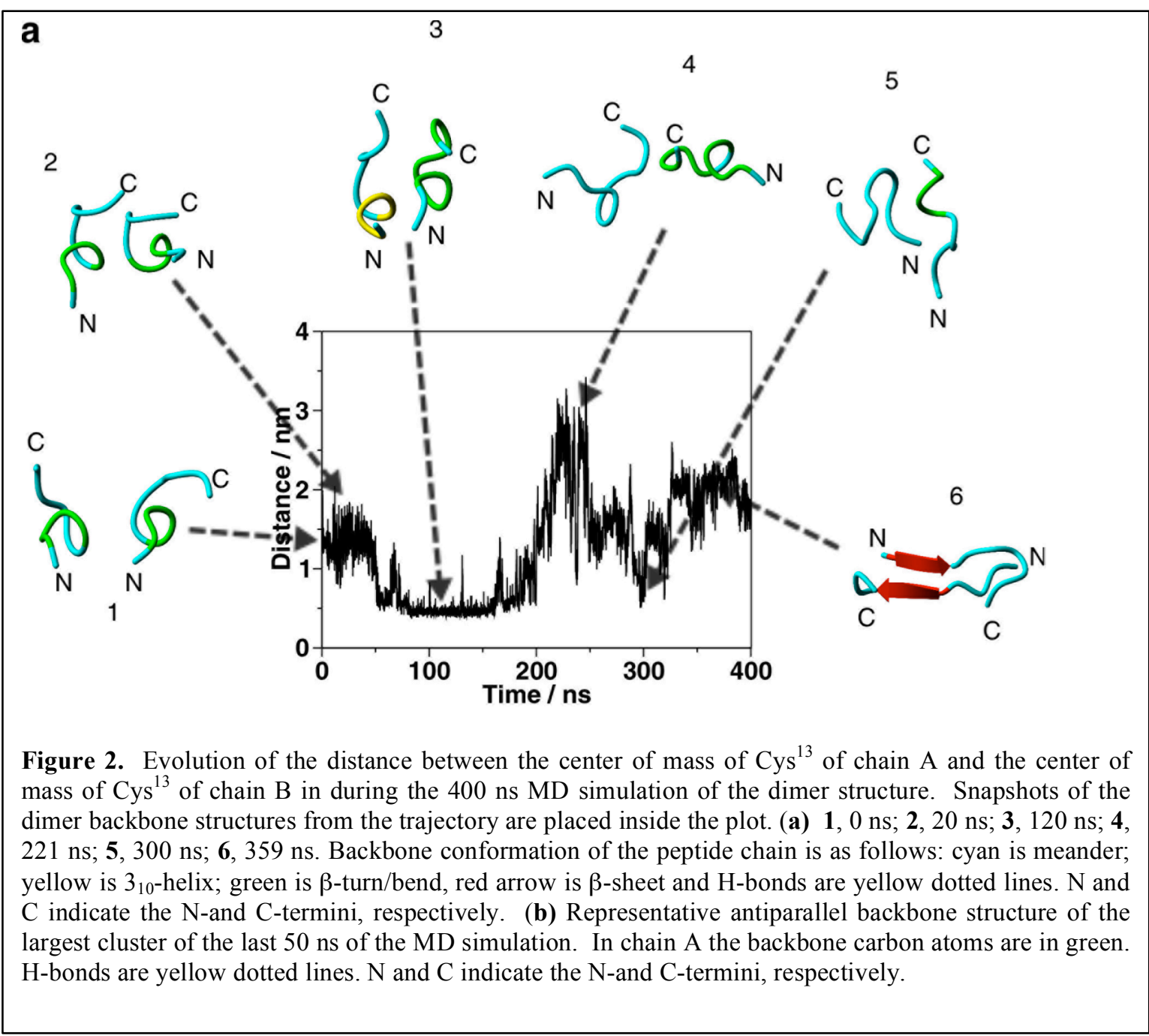

These data show that initially unfolded monomers (snapshot 1) upon interactions move relatively randomly remaining the unfolded conformation (snapshots 2-5) until they orient in antiparallel orientation and form an antiparallel $\beta$-sheet conformation shown in snapshot 6 with red arrows. Thus MD simulations revealed that the monomers change dramatically the conformation when the tight dimer is formed. Moreover, this conformation remain stable over more extended simulation time suggesting that compared to other conformations adopted by the dimer this one containing a $\beta$-sheet conformation in an antiparallel orientation of the chains forms a stable dimer structure.

The steered MD simulation was applied to analyze the rupture process for the dimer. The results demonstrate that monomers under the applied force undergo structural transition, which is accompanied by the sharp rupture of the dimer, which is in perfect agreement with experimental results. 
In conclusion, the computational analysis not only fully confirmed our model on the stabilization of misfolded state by the peptide dimerization but also provided structural details for the misfolded conformations of amyloid peptide.

Specific Aim 2 To characterize interactions leading to protein misfolding accompanied by formation of toxic aggregated morphologies (collaboration with C. Rochet, Purdue University)

The major efforts of our studies under this aim was focused on characterization of $\alpha$-Syn and self-assembly pathways resulting in the formation of membrane-bound, potentially neurotoxic $\alpha$-Syn oligomers. These oligomers are thought to play a role in neurodegeneration in the brains of patients with Parkinson's disease (PD).

During the period of support, we have made excellent progress in characterizing parameters involved in $\alpha$-Syn aggregation in the presence of phospholipid membranes. We have used epi-fluorescence microscopy of supported bilayers to study $\alpha-S y n-m e m b r a n e$ interactions. We find that membranes enriched with anionic lipids induce a clustering of fluorescently labeled $\alpha$ Syn applied to the bilayer. In addition, $\alpha$ Syn accumulations with different stabilities are formed on a lipid bilayer at $\mathrm{pH} 5.0$ versus 7.4, presumably because the C-domain is substantially less charged at the lower $\mathrm{pH}$. We also find that $\alpha$ Syn triggers a redistribution of membrane lipids into 'tubule'-like structures when added to bilayers with relatively low levels $(<30 \mathrm{~mol} \%)$ of anionic phospholipid. Presumably, $\alpha$ Syn -induced tubule formation results from a perturbation of membrane curvature as exposed, unstructured, hydrophobic regions of the protein penetrate the bilayer.

Collectively, these data suggest that (i) phospholipid membranes enriched with anionic phospholipids promote the conversion of $\alpha$-Syn to membrane-bound, potentially neurotoxic oligomers; (ii) $\alpha$-Syn may form different membrane-bound assemblies in intracellular compartments with different $\mathrm{pH}$ values; and (iii) $\alpha$-Syn may elicit neurotoxicity by causing an excess of membrane tubule formation, with potentially negative consequences to membrane integrity or to cellular processes such as membrane trafficking and endocytosis.

In other work, we have begun to characterize structure-function relationships underlying

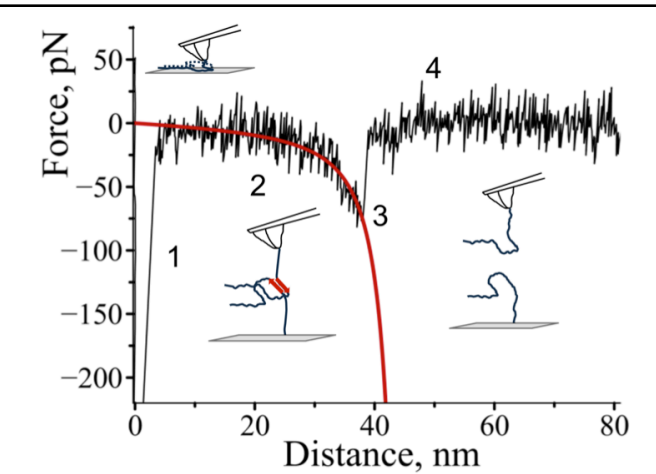

Figure 3. Typical regions on the force-distance curves: (1) an adhesion peak due to shortrange non-specific interactions between the tip and the surface, (2) gradual increase in force characteristic of polymer stretching, (3) complete rupture and (4) region where tip comes free from the surface. the formation of potentially toxic $\alpha$-Syn oligomers at the membrane surface. Wild-type $\alpha$-Syn, 'del2' (an engineered mutant missing the first two $\mathrm{N}$ terminal lysine-rich repeats), and 'plus2' (a mutant with two additional copies of repeats 1 and 2) were incubated with phospholipid vesicles for $5 \mathrm{~d}$ at 37 ${ }^{\circ} \mathrm{C}$. Phospholipid-bound and unbound proteins were separated by lipid flotation, and the fractions were analyzed via Western blotting. We found that del2 (previously shown to have a low $\alpha$-helical propensity but a high $\beta$-sheet propensity) had the most pronounced ability to form SDS-resistant aggregates in terms of both the rate and extent of self-assembly. In contrast, plus2 (previously shown to have a high a high $\alpha$-helical propensity but a low $\beta$-sheet propensity) formed membrane-bound oligomers with markedly reduced SDS resistance, 
whereas wild-type $\alpha$ Syn exhibited an intermediate ability to aggregate at the membrane surface. These data suggest that membrane-bound $\alpha$ Syn must adopt a conformation enriched with $\beta$-sheet structure rather than extensive $\alpha$-helical structure to form SDS-resistant aggregates (too much $\alpha$ helix may disfavor conformational changes required for extensive self-assembly).

In other studies, we have developed an innovative FLIM-FRET method to monitor $\alpha$-Syn aggregation in neuronal cells, in collaboration with Dr. Joseph Irudayaraj (Purdue University). Using this method, we have found that $\alpha$-Syn undergoes self-assembly and is recruited into inclusions in cells exposed to rotenone, a PD-related insult that triggers oxidative stress via inhibition of mitochondrial complex I. We are now poised to determine whether $\alpha$ Syn variants with different secondary structure propensities (e.g. wild-type, del2, plus2) have different abilities to form intracellular aggregates using the FLIM-FRET approach. Finally, we have shown that the addictive drug methamphetamine (METH), which elicits neurotoxicity similar to the neurodegenerative phenotype of PD, induces the formation of 'aggresomes' (protein inclusions similar to Lewy bodies) in cellular models of PD. Our next steps will be to monitor the effects of METH on the aggregation of different $\alpha$-Syn variants using the FLIMFRET approach described above (i.e. by exposing cells expressing the $\alpha$-Syn variants with METH in place of rotenone). We will also monitor conformational changes associated with the aggregation of pyrene-labeled $\alpha$-Syn variants at the membrane surface via fluorescence spectroscopy and fluorescence lifetime measurements, as outlined in last year's report.
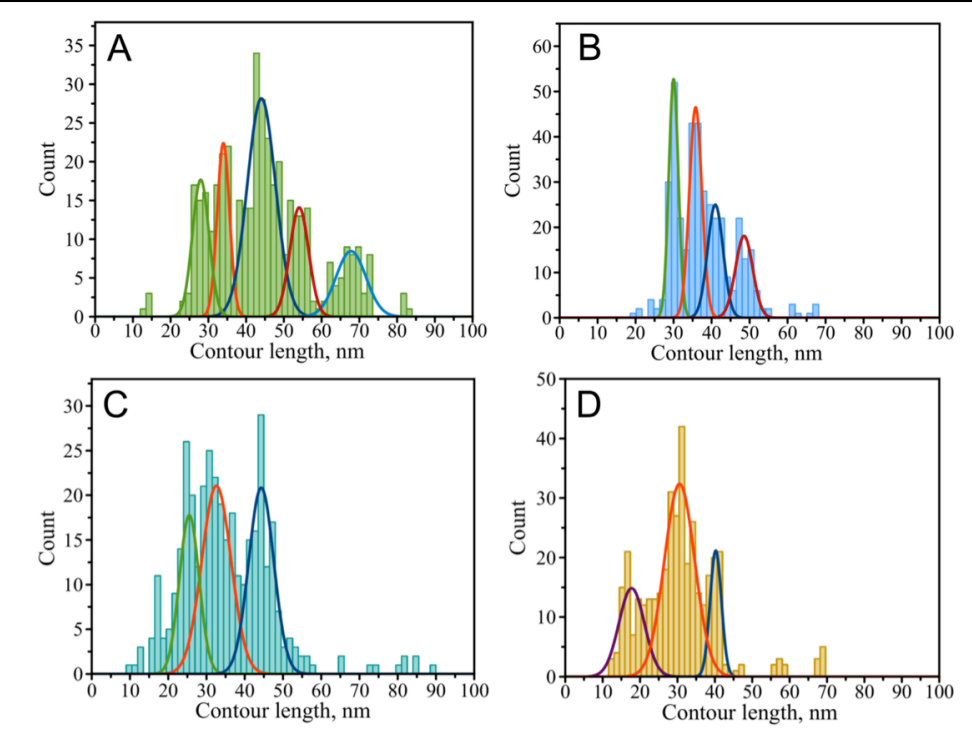

Figure 4. Contour length distribution for alpha-synuclein variants group B force-distance curves with single rupture. A) wild type (with maxima at 28, 34, 44, 54 and $68 \mathrm{~nm}$ ), B) A30P mutant (with maxima at $30,36,41$ and $49 \mathrm{~nm}$ ), C) A53T mutant (with maxima at 26, 33 and $44 \mathrm{~nm}$ ),D) E46K mutant (with maxima at 17, 31 and $40 \mathrm{~nm}$ ).
To characterize effect of familial mutations of $\alpha$ Syn on its misfolding we employed AFM force spectroscopy approach in which position of the rupture event was used as quantitative measure for the effect of the mutations. Fig. 3 explains the approach. $\alpha$-Syn molecules were covalently attached to AFM probe and mica surface via maleimide - cysteine coupling for the force spectroscopy experiments. The cysteinemaleimide coupling chemistry developed under this grant ensured very site-specific immobilization of the protein on the AFM substrate and tip surfaces required for quantitative analysis of the rupture lengths $[1,8,9]$.

\section{Interactions}

between

covalently attached molecules were probed in multiple approach-retraction cycle on various positions on the surface. If no stable dimeric contact is formed the retraction of the tip does not detect any rupture event. However, a stable dimeric contact produces a specific rupture signature in the force-distance curve. The beginning of such a force curve usually contains an adhesion peak (1) due to short-range non-specific interactions between the tip and the surface (illustrated 
in Figure 3). The adhesion peak is followed by a complete rupture event (3) where the tip comes free from the surface (4). The rupture event is preceded by a part characteristic of the stretching of polymer linkers (2). The formation of dimeric contact makes it possible to observe fine features of $\alpha$-Syn dimeric structure and conformational characteristics of misfolded proteins. Notably, the same predominant interactions were also found in $\alpha$-Syn at neutral $\mathrm{pH}$ in the presence of spermidine [9].

We applied our strategy to probe interactions between pathological single point mutants which correlate with familial early onset Parkinson's disease A30P, A53T and E46K. Results of force-distance curve analysis for these mutants are assembled in Fig. 3. Although, the distributions of contour length are quite broad, they appear simpler with fewer peaks present and slightly narrower than for WT (Figure 3, A-D). The distributions reveal different patterns for each $\alpha$-Syn variant. Similar to WT, mutants also show several peaks in $\mathrm{L}_{\mathrm{C}}$ distribution suggesting the existence of more than one conformation within the dimer. Four prominent peaks are clearly seen for A30P mutant with maxima of Gaussians at 30, 36, 41 and $49 \mathrm{~nm}$ (Fig. 3, B). Both A53T and E46K mutants had their contour length distributions skewed to shorter $\mathrm{L}_{\mathrm{C}}$ values as compared to both WT and A30P (Fig. 3 C,D). Majority of the rupture events for all $\alpha$-Syn variants appear within the range from $\sim 26 \mathrm{~nm}$ to $\sim 50 \mathrm{~nm}$. Although, there were other interactions observed at longer and shorter distances, they had lower probability than the peaks in the $26-50 \mathrm{~nm}$ range. Moreover, other peaks outside this region are not present for all the variants. For example, the shortest peak observed for WT at $28 \mathrm{~nm}$ is not present in the histogram for A30P. Also the longest peak at $68 \mathrm{~nm}$ does not have a counterpart in histograms for the mutants suggesting that this interaction is specific only for WT.

Our data demonstrate that, the pathogenic mutations A30P, E46K and A53T do not increase the propensity of $\alpha$-Syn to misfold but rather affect conformational preferences of $\alpha$-Syn molecule. We noted the direct effect of mutations that destabilize interactions of the regions located close to the position of mutations. Indirect effect of mutations is manifested in promoting interactions between segments located within $\mathrm{C}$-terminus distantly from the mutation sites. Additionally, E46K and A53T mutations increase propensity of dimerization via simultaneous interactions of multiple segments while A30P mutant prefers single type interactions compare to WT. We hypothesize that such differences in interacting patterns define aggregation pathway of the protein.

Thus the developed AFM probing approach reveals structural differences in these variants of $\alpha-$ Syn. Additionally, these studies allowed us to build the model for $\alpha-$ Syn structure at transient state that prone to the disease development.

Aim 3: To design innovative nanotechnology tools for the control of intracellular protein misfolding and aggregation processes.

Given the high sensitivity of the force probing approach, we worked on the development of this approach as a tool for detecting interaction of amyloid proteins with small molecules mediating the protein misfolding. The molecules preventing misfolding can be candidates for drugs. Moreover, the AFM force spectroscopy is a quantitative tool enabling one to arrange such candidates according to their anti misfolding activity. On another side, metabolites can increase the proteins propensity to misfold, so AFM would be a tool for identifying these metabolites and searching for their agonists. This ability of AFM has been tested on a several systems.

Using the approach schematically shown in Fig. 3 and $\alpha-$ Syn as a test system [9], we found that at $\mathrm{pH} 7.0$, where $\alpha$-Syn is randomly coiled, there are no detectable misfolding events. 
However, such cations as $\mathrm{Al}^{3+}$ or $\mathrm{Zn}^{2+}$ significantly enhance the interaction of the proteins qualitatively similar to the $\alpha$-synuclein misfolding induced by low $\mathrm{pH}$. The analysis of contour length suggests that binding of the ions change conformations of misfolded $\alpha$-Syn and the target is C-terminal end of the protein.

We mentioned above the role of metabolites of protein misfolding. We applied AFM force spectroscopy to characterize the effect of polyamines on $\alpha$-Syn misfolding. Reduced activity of the catabolic enzyme SAT1 increases cellular levels of higher order polyamines (spermine/spermidine) which in turn enhances toxicity of $\alpha$-Syn. These results suggest that polyamines in a cellular context are directly involved in PD pathology at physiological concentrations [10]. Consequently, understanding the structural basis of monomeric $\alpha$-Syn and its self-association in the presence of polyamines is of particular importance. Probing of $\alpha$-Syn interactions at neutral $\mathrm{pH}$ (PBS buffer) did not reveal rupture events suggesting that no stable dimeric contact is formed between alpha-synuclein molecules at these conditions. This observation is consistent with the above mentioned force spectroscopy studies. Next, we repeated probing experiments in the presence of $5 \mathrm{mM}$ spermidine. The effect of spermidine on the appearance of the force-distance curves was dramatic. For WT $\alpha$-Syn the yield of measurable specific rupture events was $1.8 \%$. We performed a similar study with a single point mutant A30P of a-Syn for which an early onset of PD was observed [11]. Similar to WT $\alpha$-Syn, we did not

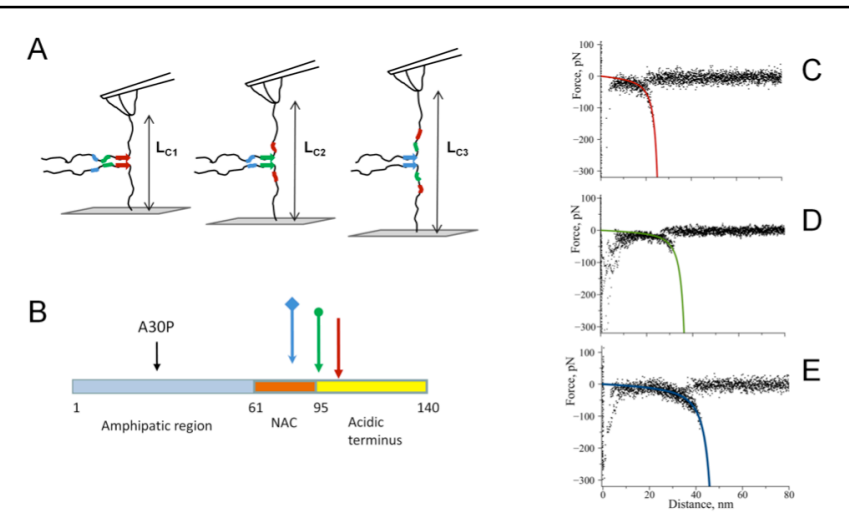

Figure 5. Interaction model of alpha-synuclein molecules. The model describes three major peaks in the contour length histograms as identical sites in each monomer interacting with each other with the formation of a dimer. A) Position of the interacting site further from the C-terminus (point of attachment) results in longer contour length value. B) Positions at the beginning of the detected interaction sites. Colored arrows correspond to three detected interaction sites schematically shown in A), and the black arrow shows the position of the A30P mutation in alpha-synuclein; C), D) and E) superposition of representative force-distance curves for the detected rupture events corresponding to $\mathrm{L}_{\mathrm{C}_{1}}, \mathrm{~L}_{\mathrm{C} 2}$ and $\mathrm{L}_{\mathrm{C} 3}$, red, green and blue lines on the graphs are WLC curves calculated with Lc values . observe any specific interactions for A30P in PBS without spermidine while in the presence of $5 \mathrm{mM}$ spermidine specific rupture events were observed. The yield of rupture events was $1.9 \%$ and it is comparable to the yield measured for WT indicating that the effect of spermidine for both WT and A30P mutant is rather similar.

The rupture events appear at a specific distance on the force-distance curve and the experimentally measured distributions of contour lengths for both proteins have clear maxima suggesting the existence of preferred interactions. Each maximum on the distribution corresponds to a certain length of stretchable, free C-terminal regions of interacting $\alpha$-Syn molecules.

Figure 5 schematically shows the model of interactions between aSyn molecules. The positions of three main peaks, $p 1, p 2$ and $p 3$, correspond to $L_{C 1}, L_{C 2}$ and $L_{C 3}$ values on the

contour length distribution. The analysis of the contour lengths suggested that peak $p 3$ with $L_{C 3}=$ $47 \mathrm{~nm}$ corresponded to lengths of two extended C-terminal parts of the protein $47 \mathrm{~nm}-3 \mathrm{~nm}$ (length of 2 MAS linkers) $=44 \mathrm{~nm}$ or $22 \mathrm{~nm}$ per one C-terminus. Given the interaminoacid 
distance $(0.34 \mathrm{~nm})$ this value leads to the C-terminus length of 65 aa. The position of the interacting region is then $140-65=75$ aa.

In summary, the use of the developed methodology led to novel findings that the very first steps of $\alpha$-Syn self-assembly are severely affected by the presence of the cellular polyamine spermidine. Our data suggest that the presence of spermidine at concentrations comparable to physiological ones induces conformational changes in $\alpha$-Syn producing a misfolded state of the $\alpha$-Syn monomer. Therefore, in addition to their normal cellular functions, biogenic polyamines may also have a pathogenic role initializing aggregation of $\alpha$-Syn. Misfolded states of $\alpha$-Syn formed in the presence of spermidine are more prone to undergo self-assembly than the normal state of the molecule as detected by single-molecule force spectroscopy. Importantly, we have found that more than one segment within the protein molecule might be responsible for the initial association of $\alpha$-Syn into dimers and possibly into higher-order oligomers and fibrils. This finding suggests that even the first step of $\alpha$-Syn self-assembly (dimerization) possesses some degree of heterogeneity. We hypothesize that these different misfolded conformations can lead to different types of oligomers and define the aggregation pathway. The marked differences in the misfolding patterns between WT $\alpha$-Syn and A30P mutant might be responsible for the higher propensity of the mutant to aggregate and cause early-onset PD.

We also applied our approach to understanding the mechanism by which $\mathrm{Cu}^{2+}$ influence the development of $\mathrm{AD}$. Several lines of evidence suggest that $\mathrm{Cu}^{2+}$ cations play an important role in the aggregation of $\mathrm{A} \beta 42$ both in vitro and in vivo. For example, a prominent characteristic of $\mathrm{AD}$ is altered $\mathrm{Cu}^{2+}$ concentrations in the brain and disrupted $\mathrm{Cu}^{2+}$ homeostasis [12]. $\mathrm{Cu}^{2+}$ ions are found concentrated within senile plaques of $\mathrm{AD}$ patients directly bound to $\mathrm{A} B$ with a picomolar affinity [13]. The concentration of $\mathrm{Cu}^{2+}$ within the senile plaques of $\mathrm{AD}$ patients is 26 times higher than within the extracellular space of healthy individuals [14]. Aß plaques are therefore considered a metal "sink" [15]. Copper in a high-cholesterol diet induces amyloid plaque formation and learning deficits in a rabbit model of AD [16]. Trace amounts of metal cations initiate and promote $\mathrm{Ab}$ aggregation [17, 18]. Moreover, metal cations are believed to contribute to AD pathogenesis by causing oxidative stress, which could lead to the dysfunction or death of neuronal cells [19]; [20]. One of the prevailing underlying mechanisms of AD etiology is the metal-triggering hypothesis [21,22].

Despite the fact that the effects of $\mathrm{Cu}^{2+}$ cations on $\mathrm{A} B$ aggregation have been extensively investigated, the underlying mechanism controlling aggregation remains elusive. One of the main challenges is obtaining detailed $\mathrm{Cu}^{2+}-\mathrm{A} \beta 42$ interaction information during the earliest stage of aggregation, especially in the dimerization phase, because oligomers are transient states not amenable to traditional visualization techniques. In addition, the ability of $\mathrm{Cu}^{2+}$ cations to promote the growth of fibrils needs to be verified. Therefore, a thorough study capable of probing transient states of $\mathrm{Cu}^{2+}-\mathrm{A} \beta 42$ interactions at the single molecule level would be significant.

We applied single molecule AFM force spectroscopy to elucidate the role of $\mathrm{Cu}^{2+}$ cations on interpeptide interactions. The effect of $\mathrm{Cu}^{2+}$ cations on the $\mathrm{A} \beta 42$ interaction was investigated by SMFS at $\mathrm{pH}$ values of 7.4, 6.8, 6.0, 5.0 and 4.0. An overlap of all raw force curves obtained in the absence and presence of $\mathrm{Cu}^{2+}$ cations and at the physiological condition, $\mathrm{pH} 7.4$, is shown in the left column of Fig. 6.

Clustered data points at certain rupture lengths and rupture forces represent visual presentations of the overlay of multiple rupture events and provide a clear comparison between the presence and absence of $\mathrm{Cu}^{2+}$ cations. 


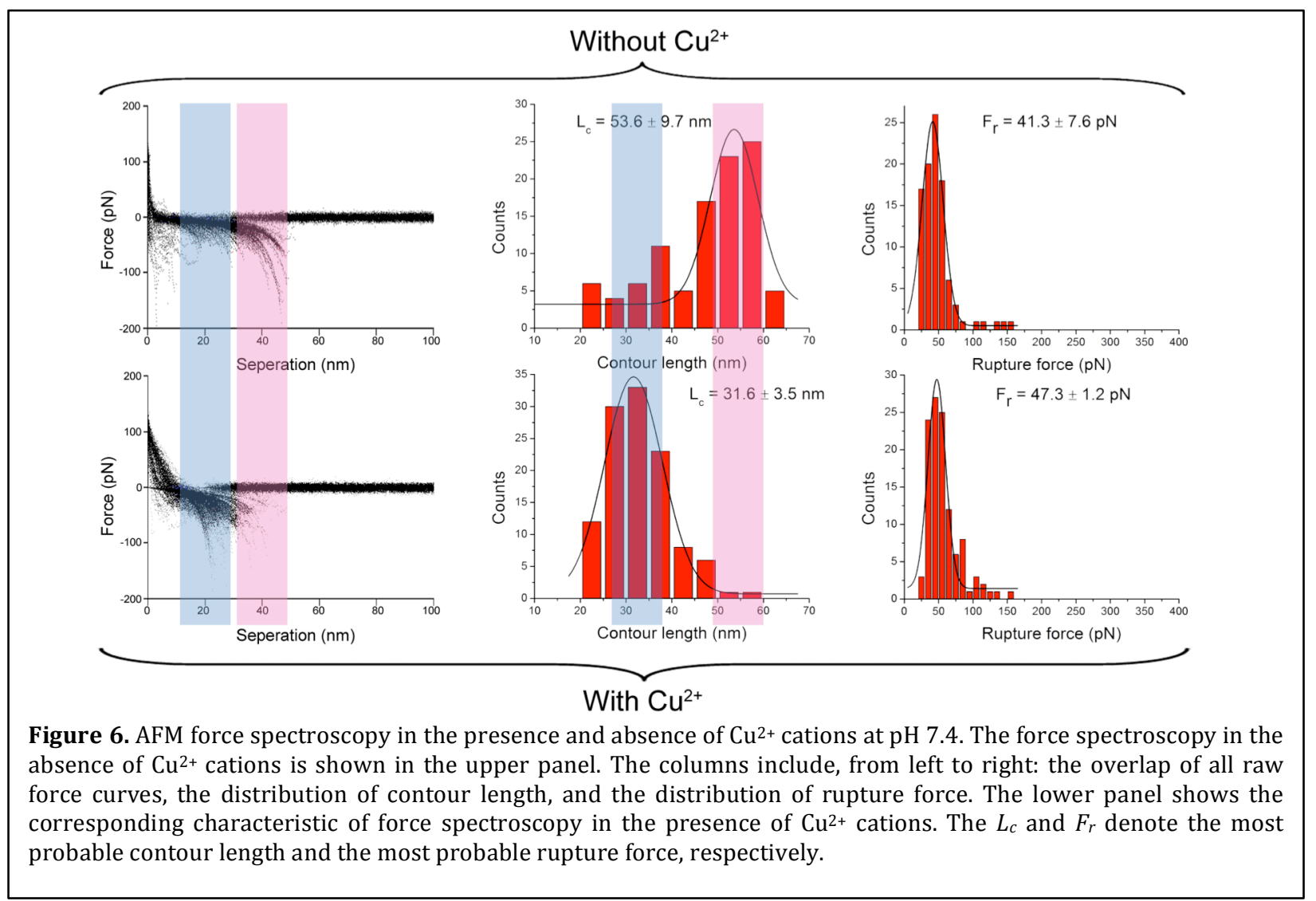

Major differences between these two types of experiments are highlighted with colored light pink or light blue vertical bands. The contour length analysis as described above showed that $\mathrm{Cu}^{2+}$ cations induce $\mathrm{A} \beta 42$ peptide structural changes resulting in N-termini interactions within the dimers. $\mathrm{Cu}^{2+}$ cations also stabilize the dimers. No effects of $\mathrm{Cu}^{2+}$ cations on $\mathrm{A} \beta-\mathrm{A} \beta$ interactions were observed at $\mathrm{pH} 4.0$, suggesting that peptide protonation changes the peptide-cation interaction. The effect of $\mathrm{Cu}^{2+}$ cations on later stages of $A B$ aggregation was studied by AFM topographic images. The results demonstrate that substoichiometric $\mathrm{Cu}^{2+}$ cations accelerate the formation of fibrils at $\mathrm{pH} 7.4$ and 5.0, whereas no effect of $\mathrm{Cu}^{2+}$ cations was observed at $\mathrm{pH}$ 4.0. Taken together, the combined AFM force spectroscopy and imaging analyses demonstrate that $\mathrm{Cu}^{2+}$ cations promote both the initial and the elongation stages of $\mathrm{A} \beta$ aggregation, but protein protonation diminishes the effect of $\mathrm{Cu}^{2+}$.

\section{Methodology development for AFM force spectroscopy.}

The experimental approaches described above depend on the availability of the surface chemistry enabling reliable and controlled immobilization of molecules of interest on the substrate and surfaces. We worked with our collaborator Dr. J. Keana on the development of the silatrane surface chemistry. One of the specific goals in this area was the development of novel flexible spacer for the force spectroscopy.

Flexible spacers between the molecules and the surface play multiple roles. First, they provide mobility for the tethered molecule in the space required for proper orientation of the molecules for their interaction as the molecules approach one another. Second, polymeric tethers 
separate the rupture event of interest from any non-specific, short-range tip-surface interactions, thus aiding in the identification of particular rupture events while reducing the effects of mechanical noise. Third, the extension of the spacer to a definite length is a specific signature on the force-distance curve, allowing one to unambiguously identify the single rupture force event. The availability of functionalized-PEG polymers facilitated their use as convenient polymeric spacers (reviewed in [23]). However, heterogeneity in size of commercially available, bifunctionalized PEGs leads to a variability in the separation distance for the rupture event, which is a serious complication in the analysis of the AFM force spectroscopy data [24-26]. Based on these findings, we proposed in the application the development of a novel class of functionalized flexible linkers for AFM force spectroscopy studies. The linkers will be used as tethers and will have the following three major characteristics: (1) well-defined lengths, (2) different tether compositions, and (3) available with different functionalized groups allowing for reliable covalent surface immobilization.

\section{Phosphoramidites for DNA Synthesis and the Feasibility of Synthesis of Novel Flexible Tethers}

The proposed likers are based on the synthesis of flexible polymers with the use of the nonnucleoside PA spacers and a standard oligonucleotide synthesis to grow polymeric chain with the desired length. The rationale for this approach is supported by the fact that PA spacers can be incorporated into a defined site within synthetic oligonucleotides [27-29]. Moreover, a standard DNA synthesis methodology can be applied. Note also that the PA spacers are commercially available (Glen Research, Sterling, VA and TriLink BioTechnologies, San Diego, CA).

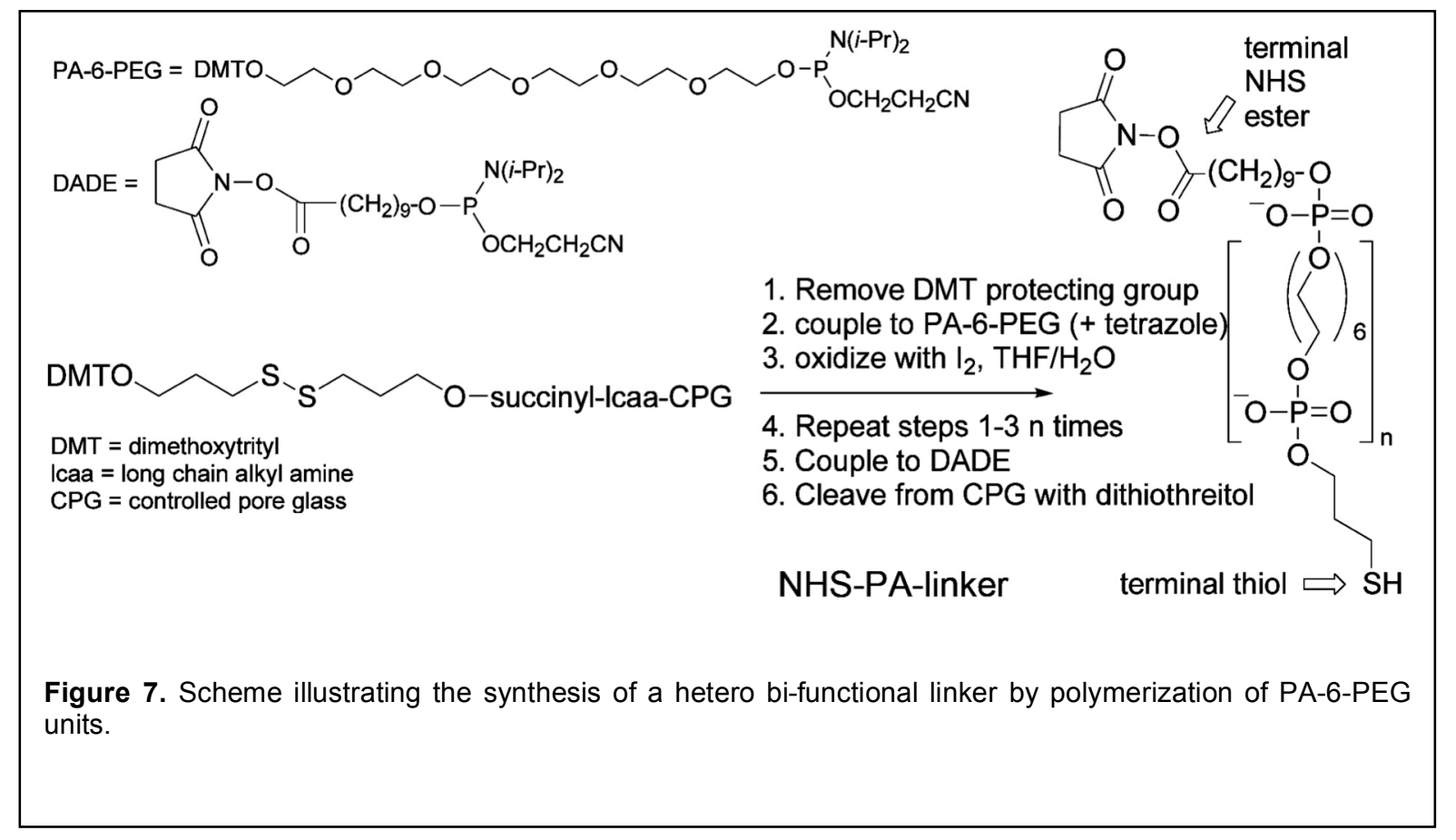

We have recently tested this idea using the commercially available PA and phosphoramidite DNA polymerization chemistry [30].

The overall idea is illustrated in Fig. 7 in which synthesis of the PA tether terminated with SH and N-hydroxysuccinimide (NHS) groups is demonstrated. We used the non-nucleoside spacer PA-6-PEG (18-O-dimethoxytrityl-hexaethyleneglycol-1-[2-cyanoethyl)- $N, N$-diisopropyl)]- 
phosphoramidite (TriLink BioTechnologies). One end of this spacer was protected with a dimethoxytrityl (DMT) group and the other end was terminated with a cyanoethylphosphoramidite (CEPA) group, as required for phosphoramidite polymerization chemistry. The synthesis was performed in a DNA synthesizer and consists of three primary steps; it begins with the DMT-protected disulfide immobilized on CPG glass beads. In the first step, the DMT protecting group is removed, and in the second step, the free hydroxyl $(\mathrm{OH})$ group is coupled to the phosphoramidite PA-6-PEG. In the third step, oxidation produces the stable phosphate linkage. Steps 1-3 are repeated as required by the experiment. After the final "step 1" is completed, the terminal $\mathrm{OH}$ group is coupled to the phosphoramidite DADE, which contains a terminal NHS ester group.

Steps 1-3 were repeated twelve times to finally result in a linker with 72 ethylene glycol units (i.e. $\left.(\mathrm{PA}-\mathrm{PEG}-6)_{12}\right)$ :

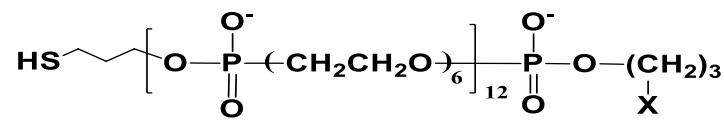

This length of a flexible PA molecule is similar to the size of PEG linkers typically used in force spectroscopy studies. Similarly, the tether with a terminal NHS group was synthesized; it remained bound to the glass beads via hydrolysable disulfide bond (SS-bond). We tested this tether in force spectroscopy experiments to probe the interaction between complementary ssDNA oligonucleotides (20-mer): ssDNA1 and ssDNA2. The flow chart for the immobilization of DNA molecules on the mica surface and AFM tip is shown below in Fig. 8.

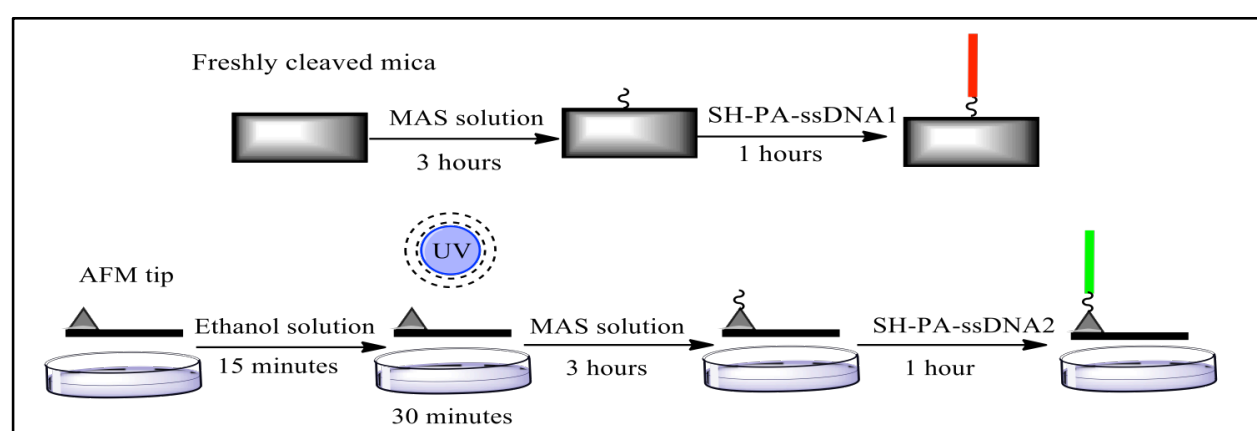

Figure 8. Flow chart for the immobilization procedure of complementary DNA oligonucleotides on the mica surface and AFM tip with the use of (PA-PEG-6) ${ }_{12}$ tether.
The NHS (PAPEG-6) 12 tether bound to the glass beads was first reacted with the two complimentary ssDNA that had an amine group at 5'ends. The reaction took place in DMSO to prevent the hydrolysis of the NHS group. The PA-ssDNA was cleaved from glass bead by $100 \mathrm{mM}$ DTT at $\mathrm{pH} 8.5$ buffer, and the coupling reaction was monitored with gel electrophoresis.

The mica sheets were cut into $1.5 \mathrm{~cm} \times 1.5 \mathrm{~cm}$ squares, cleaved, and treated with MAS for $3 \mathrm{~h}$ followed by the incubation in the solution of PA-ssDNA1 for $1 \mathrm{~h}$. DTT was extracted from the PA-ssDNA1 solution by ethylacetate before the use. Silicon nitride $\left(\mathrm{Si}_{3} \mathrm{~N}_{4}\right)$ AFM tips were left in ethanol for $15 \mathrm{~min}$, rinsed with water, dried out with stream argon, and treated by $254 \mathrm{~nm}$ UV for $30 \mathrm{~min}$. The AFM tips were immersed in MAS solution for $3 \mathrm{~h}$ followed by the binding of PA-ssDNA2 for $1 \mathrm{~h}$. After rinsing, the unreacted maleimide moieties on the mica surface and AFM probe were blocked by treatment with $10 \mathrm{mM} \beta$-mercaptoethanol for $10 \mathrm{~min}$. 


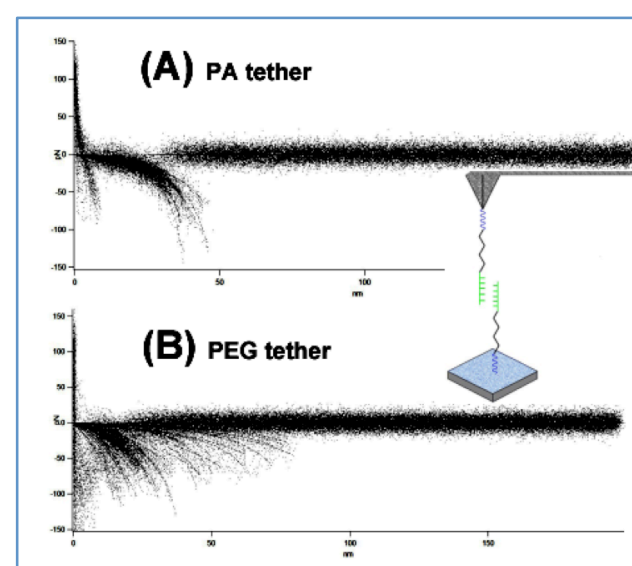

Figure 9. The overlay of the force curves for the interaction of 20 nt DNA obtained with the use of PA-tether $(A)$ and PEG tether (B). Inset illustrates schematically the AFM probing experiment.
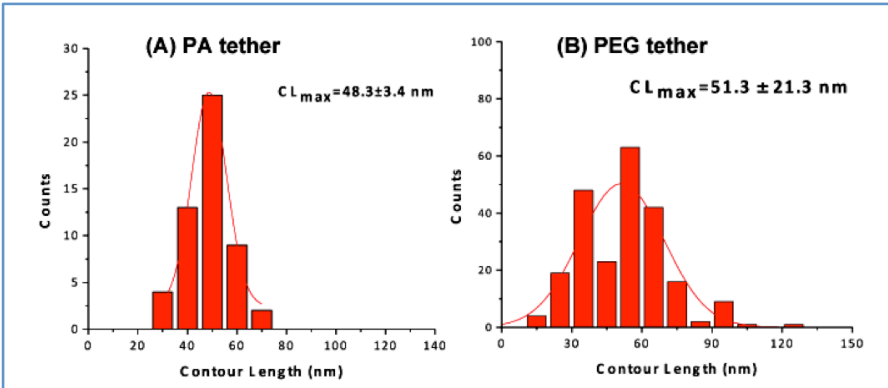

Figure 10. The histogram of the contour lengths distribution for the force spectroscopy experiments with the use of PAtether (A) and PEG tether (B).

In parallel, ssDNA 2 were immobilized on the tip with the use of PEG tether.

The single-molecule force measurement was performed at $\mathrm{pH} 7.4 \mathrm{PBS}$ buffer. A $100 \mathrm{pN}$ trigger force was applied to the probe. The spring constants of the probes were between $0.015-0.030 \mathrm{~N} / \mathrm{m}$. The retract velocity was

$600 \mathrm{~nm} / \mathrm{s}$. Igor Pro 5.03 software was used for data analysis. Contour length, rupture force, and persistence length were obtained from rupture curves fitted by the WLC model.

Figure 9 is the overlay of more than 100 force curves acquired in these experiments with the use of a PA tether. The curves are grouped around the rupture length of $\sim 35 \mathrm{~nm}$. Similar datasets for probing experiments with the use of PEG tether are shown in Fig. 9B. The force curves are also grouped around $30 \mathrm{~nm}$ rupture length, but there are many events with larger rupture lengths that can be explained by the PEG tether heterogeneity. This is illustrated graphically in Fig. 10 in which the contour lengths distributions are shown.

Thus, these data provide the justification for the feasibility of PA tethers approach and their use for the AFM force spectroscopy experiments. The homogeneity of the tether length is a key issue in these studies. This homogenous property of the PA tether is a property that is needed for the studies described above and the data in [30] illustrate the advantages of PA linker compared to PEG one in studies protein misfolding.

\section{Combination of AFM and Raman - a novel tool for studies of protein aggregation}

The integration of AFM in spectroscopic systems opens large prospects for combining the topographic data (AFM module) with the chemical structural data provided by a spectroscopy technique. One of such spectroscopic techniques is Raman spectroscopy, which allows one to characterize the sample structure without labeling the sample. A breakthrough in this area of the instrument's development enabled the successful integration of AFM and Raman spectroscopy. 


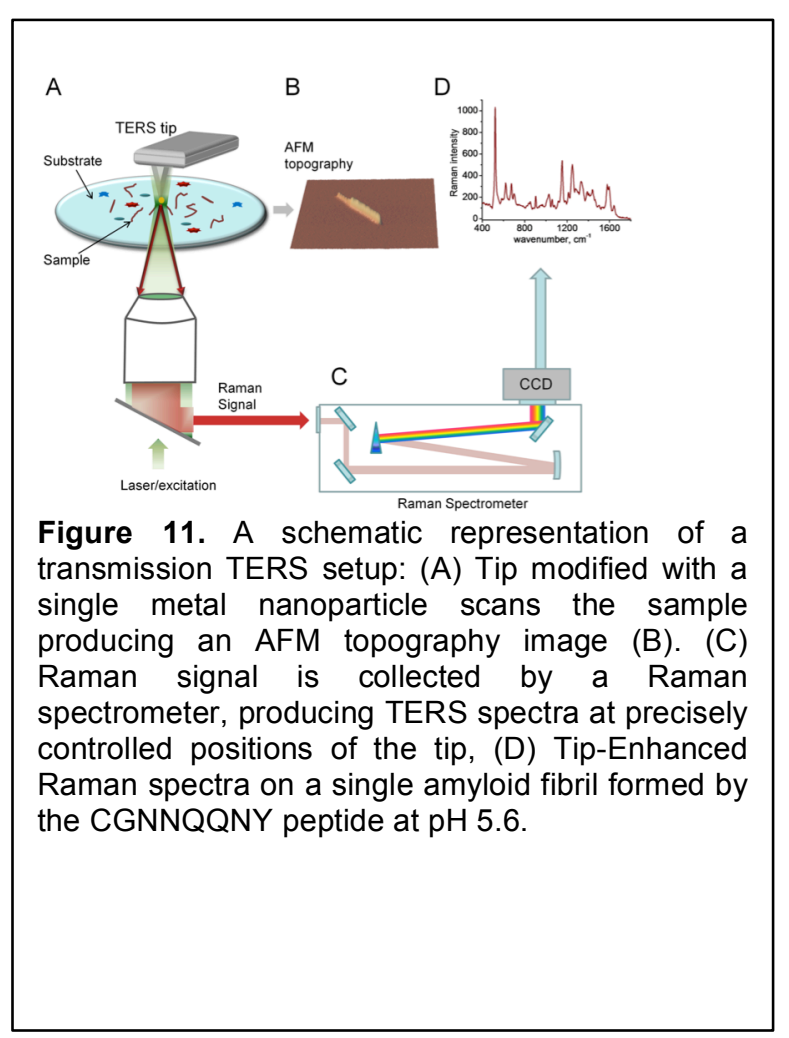

.Figure 11 shows schematic representation of the integrated AFM-Raman instrument utilizing TERS in transmission setup. The TERS inherent capability is provided by the nanoscale, metallic tip. Importantly, the electromagnetic field is enhanced locally by the metal nanoparticle due to plasmon resonance in the metal so that the enhancement factors can reach several orders of magnitude.
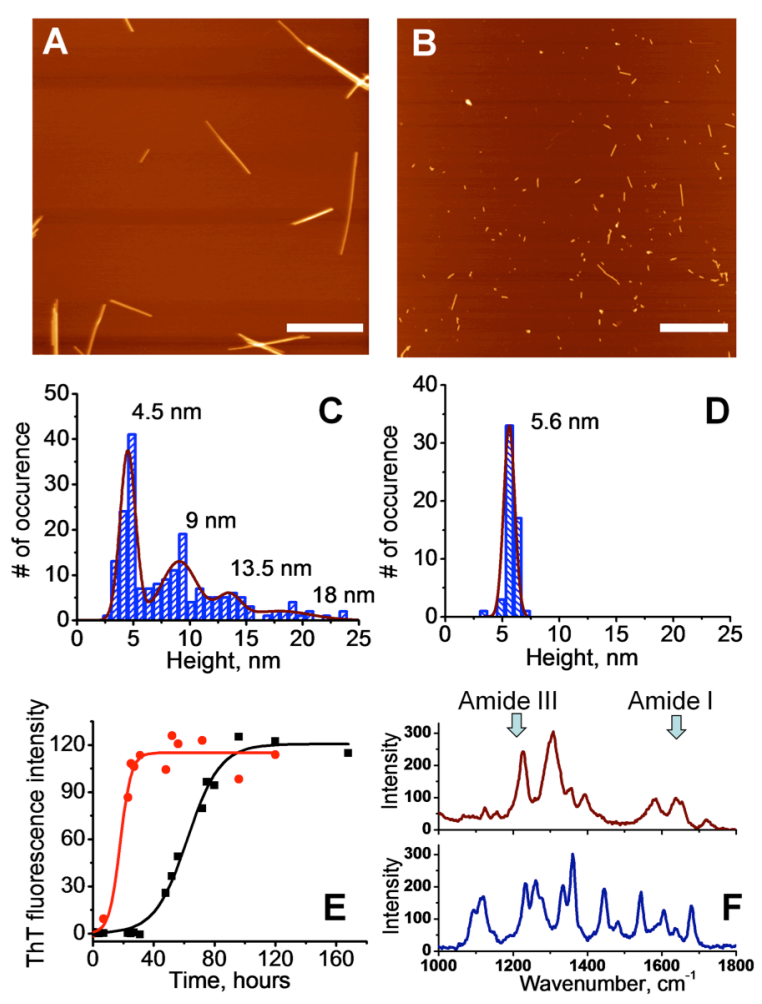

Figure 12. AFM images of fibrils formed by CGNNQQNY peptide from Sup35 yeast prion protein dissolved in $\mathrm{A}$ ) water $(\mathrm{pH} 2)$ and $\mathrm{B}$ ) pH5.6. $C, D)$ height distributions of fibrils for water and $\mathrm{pH}$ 5.6 correspondingly. E) Aggregation kinetics (ThT assay) of CGNNQQNY peptide in water (black) and pH 5.6 (red). F) TERS spectra of fibrils formed by CGNNQQNY peptide from Sup35 yeast prion protein in water (top) and pH5.6 (bottom) showing different positions of amide bands characteristic of polypeptide secondary structure.

These technologies are expected to significantly impact our collaborative projects. Specifically, TERS/AFM developed for projects focused on imaging of amyloid aggregates will eventually lead to elucidating molecular mechanisms of development of Alzheimer's, Parkinson's, prion and other protein misfolding diseases. Furthermore, the new technology for the thin substrate preparation is the key for the development of a novel and sensitive tool for early cancer diagnostics.

We have recently demonstrated that the AFM/Raman method can be used to analyze structural features of protein aggregates, as illustrated in Figure 12 [31]. Two different types of fibrils formed by the CGNNQQNY peptide (fragment of Sup35 yeast prion protein) were studied; one type of fibrils was formed in water at $\mathrm{pH} \sim 2.0$ and the other type was formed in acetate buffer at pH 5.6. 
These conditions produce morphologically distinct fibrils. The fibrils formed in water were long and straight (Fig. 12A and $\mathbf{C}$ ) and tended to bundle together. Fibrils formed at $\mathrm{pH} 5.6$, on the other hand, were short with no multiple bundles observed (Fig. 12B and D). Figure 12F shows two spectra of amyloid fibrils formed by the CGNNQQNY peptide (fragment of Sup35 yeast prion protein) in water (top) and in acetate buffer - $\mathrm{pH} 5.6$ (bottom). The specific positions of the amide I and amide III bands provide information about secondary structure of the peptides that compose the fibrils. The positions of the amide bands suggest that fibrils formed at $\mathrm{pH} 5.6$ have less beta-sheet structure compared to the fibrils formed in water. For fibrils with varying secondary structures, TERS enables precise localization and assignment of different conformations, which is not amenable to other methods.

Typically, the sample topography is first acquired with the AFM module of the AFM/Raman instrument. Next, the tip is positioned in the area of interest, and Raman spectra are collected over the selected area. Figure 13 shows three Raman spectra measured $7 \mathrm{~nm}$ apart on a single fibril formed by the short CGNNQQNY peptide at $\mathrm{pH} 5.6$ [31]. It is possible to assign Raman bands corresponding to cysteine (C) and tyrosine (Y) amino acids (Figure 13) as distinct amino acids that are distinguishable by specific spectral features [32, 33]. Moreover, differences in spectra show that true nanometer resolution was achieved. Based on the intensity change of Raman signals the lateral

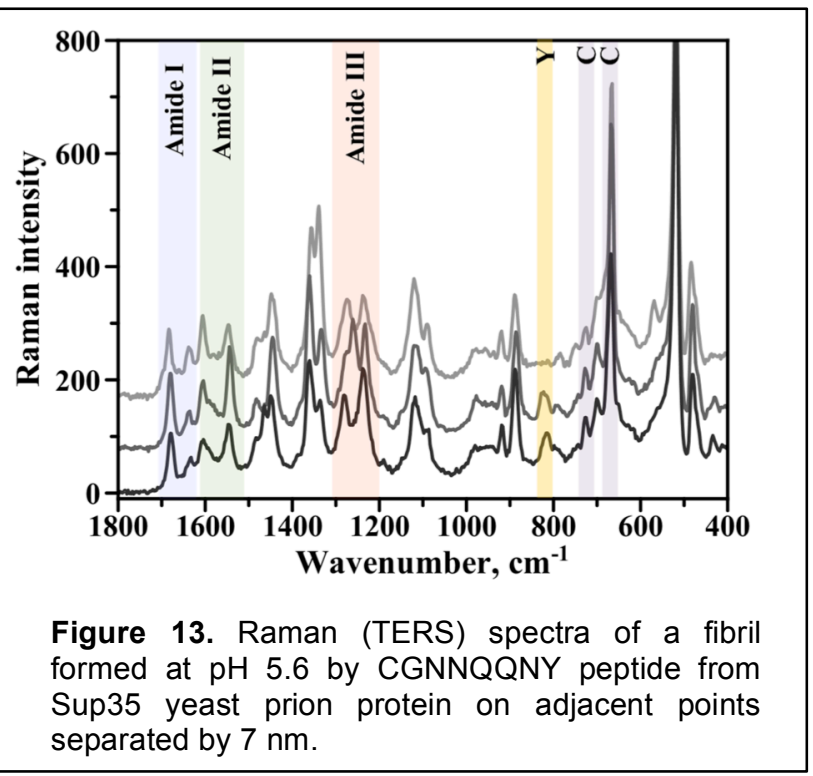
resolution was conservatively estimated to be $\sim 8.5 \mathrm{~nm}$. These novel findings demonstrate the power of TERS to provide valuable information on the structure of individual fibrils as well as on the variability of the secondary structures of peptides/proteins along the fibril. We are currently preparing a manuscript describing our findings in detail.

Therefore, a novel combined AFM and Raman method will help to overcome the limitations in the currently available methods by establishing the structure-morphology-toxicity relationship of protein aggregates, thus leading to a better understanding of prion misfolding and aggregation. The information gleaned from this technique is crucial for understanding the molecular mechanism of protein self-assembly into amyloids, which the currently available methods are not capable of providing.

In conclusion, the research supported by this award led to a number discoveries and novel technology development briefly summarized below.

- The single molecule AFM force spectroscopy studies led to the discovery that dimerization is the event triggering protein misfolding. The computational analyses supported the model and identified structural motifs stabilizing the dimers. Misfolded states of protein are transient conformations that are not amenable to traditional ensemble 
technique. With the developed approach we are able to not only identify these conformation, but also characterize those and localize protein segments involved into misfolding.

- The dimers are stable transient species facilitating further aggregation process suggesting that targeting dimers is the path for the development of diagnostic tools and treatments for the diseases. Given the fact that these species can be detected with AFM force spectroscopy, this technique is in a unique position for the drug design and development studies.

- We demonstrated by studies of a number of systems that AFM force spectroscopy approach is a valuable tool for studies of protein misfolding phenomenon.

- We also showed that the sensitivity of AFM force spectroscopy is very high. This is a single molecule probing technique enabling one to perform evaluation of small molecules in very small amounts. This unique property of the developed technology is important in the drug development studies and appropriate developments are under way.

Literature cited

1. Lyubchenko, Y.L., et al., Nanoimaging for protein misfolding diseases. Wiley Interdiscip Rev Nanomed Nanobiotechnol, 2010. 2(5): p. 526-43.

2. Bernstein, S.L., Nicholas F. Dupuis, Noel D. Lazo, Thomas Wyttenbach, Margaret M. Condron, Gal Bitan, David B. Teplow, Joan-Emma Shea, Brandon T. Ruotolo, Carol V. Robinson and Michael T. Bowers, Amyloid-b protein oligomerization and the importance of tetramers and dodecamers in the aetiology of Alzheimer's disease. Nature Chemistry, 2009. 1: p. 326-331.

3. Ono, K., M.M. Condron, and D.B. Teplow, Structure-neurotoxicity relationships of amyloid beta-protein oligomers. Proc Natl Acad Sci U S A, 2009. 106(35): p. 14745-50.

4. Shankar, G.M., et al., Biochemical and immunohistochemical analysis of an Alzheimer's disease mouse model reveals the presence of multiple cerebral Abeta assembly forms throughout life. Neurobiology of disease, 2009. 36(2): p. 293-302.

5. Shankar, G.M., et al., Natural oligomers of the Alzheimer amyloid-beta protein induce reversible synapse loss by modulating an NMDA-type glutamate receptor-dependent signaling pathway. J Neurosci, 2007. 27(11): p. 2866-75.

6. $\quad$ Yankner, B.A., T. Lu, and P. Loerch, The aging brain. Annu Rev Pathol, 2008. 3: p. 41-66.

7. He, X., J.T. Giurleo, and D.S. Talaga, Role of small oligomers on the amyloidogenic aggregation freeenergy landscape. J Mol Biol, 2009. 395(1): p. 134-54.

8. Kransnoslobodtsev, A.V., et al., Nanomedicine and protein misfolding diseases. Nanomedicine : nanotechnology, biology, and medicine, 2005. 1(4): p. 300-5.

9. Krasnoslobodtsev, A.V., et al., Effect of spermidine on misfolding and interactions of alpha-synuclein. PLoS ONE, 2012. 7(5): p. e38099.

10. Lewandowski, N.M., et al., Polyamine pathway contributes to the pathogenesis of Parkinson disease. Proceedings of the National Academy of Sciences of the United States of America, 2010. 107(39): p. 16970-5.

11. Kruger, R., et al., Ala30Pro mutation in the gene encoding alpha-synuclein in Parkinson's disease. Nature genetics, 1998. 18(2): p. 106-8.

12. Roberts, B.R., et al., The role of metallobiology and amyloid-beta peptides in Alzheimer's disease. J Neurochem, 2012. 120 Suppl 1: p. 149-66.

13. Hong, L. and J.D. Simon, Insights into the thermodynamics of copper association with amyloid-beta, alpha-synuclein and prion proteins. Metallomics, 2011. 3(3): p. 262-6.

14. Chen, W.T., et al., Distinct effects of $\mathrm{Zn} 2+, \mathrm{Cu} 2+, \mathrm{Fe} 3+$, and Al3+ on amyloid-beta stability, oligomerization, and aggregation: amyloid-beta destabilization promotes annular protofibril formation. J Biol Chem, 2011. 286(11): p. 9646-56.

15. Atwood, C.S., et al., Dramatic aggregation of Alzheimer abeta by Cu(II) is induced by conditions representing physiological acidosis. J Biol Chem, 1998. 273(21): p. 12817-26. 
16. Sparks, D.L. and B.G. Schreurs, Trace amounts of copper in water induce beta-amyloid plaques and learning deficits in a rabbit model of Alzheimer's disease. Proc Natl Acad Sci U S A, 2003. 100(19): p. 11065-9.

17. Huang, X., et al., Trace metal contamination initiates the apparent auto-aggregation, amyloidosis, and oligomerization of Alzheimer's Abeta peptides. J Biol Inorg Chem, 2004. 9(8): p. 954-60.

18. Innocenti, M., et al., Trace copper(II) or zinc(II) ions drastically modify the aggregation behavior of amyloid-beta1-42: an AFM study. J Alzheimers Dis, 2010. 19(4): p. 1323-9.

19. Jomova, K., et al., Metals, oxidative stress and neurodegenerative disorders. Mol Cell Biochem, 2010. 345(1-2): p. 91-104.

20. Barnham, K.J., C.L. Masters, and A.I. Bush, Neurodegenerative diseases and oxidative stress. Nat Rev Drug Discov, 2004. 3(3): p. 205-14.

21. Hung, Y.H., A.I. Bush, and R.A. Cherny, Copper in the brain and Alzheimer's disease. J Biol Inorg Chem, 2010. 15(1): p. 61-76.

22. Rivera-Mancia, S., et al., The transition metals copper and iron in neurodegenerative diseases. Chem Biol Interact, 2010. 186(2): p. 184-99.

23. Riener, C.K., et al., A Simple Test System for Single Molecule Recognition Force Microscopy. Analytica Chimica Acta, 2003. 479: p. 59-75.

24. Yu, J., S. Malkova, and Y.L. Lyubchenko, alpha-Synuclein misfolding: single molecule AFM force spectroscopy study. Journal of molecular biology, 2008. 384(4): p. 992-1001.

25. Kim, B.H., et al., Single-Molecule Atomic Force Microscopy Force Spectroscopy Study of Abeta-40 Interactions. Biochemistry, 2011. 50(23): p. 5154-5162.

26. Yu, J., J. Warnke, and Y.L. Lyubchenko, Nanoprobing of alpha-synuclein misfolding and aggregation with atomic force microscopy. Nanomedicine : nanotechnology, biology, and medicine, 2011. 7(2): p. 146-52.

27. Dolinnaya, N.G., et al., Oligonucleotide circularization by template-directed chemical ligation. Nucleic Acids Res, 1993. 21(23): p. 5403-7.

28. Shchepinov, M.S., et al., Oligonucleotide dendrimers: synthesis and use as polylabelled DNA probes. Nucleic Acids Res, 1997. 25(22): p. 4447-54.

29. Weston, B.F., I. Kuzmine, and C.T. Martin, Positioning of the start site in the initiation of transcription by bacteriophage T7 RNA polymerase. Journal of molecular biology, 1997. 272(1): p. 21-30.

30. Tong, Z., Andrew Mikheikin, Alexey Krasnoslobodtsev, Zhengjian Lv and Yuri L. Lyubchenko, Novel Polymer Linkers for Single Molecule AFM Force Spectroscopy. Methods, 2013. in press.

31. Krasnoslobodtsev, A.V., et al., Nanoimaging for prion related diseases. Prion, 2010. 4(4): p. 265-74.

32. Deckert-Gaudig, T. and V. Deckert, Ultraflat transparent gold nanoplates--ideal substrates for tipenhanced Raman scattering experiments. Small, 2009. 5(4): p. 432-6.

33. Deckert-Gaudig, T., E. Rauls, and V. Deckert, Aromatic Amino Acid Monolayers Sandwiched between Gold and Silver: A Combined Tip-Enhanced Raman and Theoretical Approachв 5 The Journal of Physical Chemistry C, 2009. 114(16): p. 7412-7420.

34. Yu, J., S. Malkova, and Y.L. Lyubchenko, alpha-Synuclein misfolding: single molecule AFM force spectroscopy study. J Mol Biol, 2008. 384(4): p. 992-1001.

35. Lyubchenko, Y.L. and L.S. Shlyakhtenko, AFM for analysis of structure and dynamics of DNA and protein-DNA complexes. Methods, 2009. 47(3): p. 206-13.

36. Lyubchenko, Y.L., L.S. Shlyakhtenko, and A.A. Gall, Atomic force microscopy imaging and probing of DNA, proteins, and protein DNA complexes: silatrane surface chemistry. Methods Mol Biol, 2009. 543 : p. 337-51.

37. Rubinstein, A., Y.L. Lyubchenko, and S. Sherman, Dynamic properties of $p H$-dependent structural organization of the amyloidogenic beta-protein (1-40). Prion, 2009. 3(1): p. 31-43.

38. Yu, J. and Y.L. Lyubchenko, Early stages for Parkinson's development: alpha-synuclein misfolding and aggregation. J Neuroimmune Pharmacol, 2009. 4(1): p. 10-6.

39. Feng, Y., A. Kloczkowski, and R.L. Jernigan, Potentials ' $R$ ' Us web-server for protein energy estimations with coarse-grained knowledge-based potentials. BMC bioinformatics, 2010. 11: p. 92.

40. Haque, F., et al., Adsorption of alpha-synuclein on lipid bilayers: modulating the structure and stability of protein assemblies. The journal of physical chemistry. B, 2010. 114(11): p. 4070-81. 
41. Rashin, A.A., A.H. Rashin, and R.L. Jernigan, Diversity of function-related conformational changes in proteins: coordinate uncertainty, fragment rigidity, and stability. Biochemistry, 2010. 49(27): p. 5683704.

42. Krasnoslobodtsev, A.V., B.-H. Kim, and Y.L. Lyubchenko, Nanoimaging for protein misfolding diseases. Critical role of misfolded dimers in the amyloid self-assembly. Microsc. Microanal. 17 (Suppl 2), 2011. 17(Suppl 2): p. 170-171.

43. Lyubchenko, Y.L., Preparation of DNA and nucleoprotein samples for AFM imaging. Micron, 2011. 42(2): p. 196-206.

44. Lyubchenko, Y.L., AFM imaging in liquid of DNA and protein-DNA complexes, in AFM in Liquids, A.M.a.R. Baro, R. , Editor 2011, Wiley-VCH.

45. Lyubchenko, Y.L., L.S. Shlyakhtenko, and T. Ando, Imaging of nucleic acids with atomic force microscopy. Methods, 2011. 54(2): p. 274-83.

46. Pandey, A.P., et al., alpha-Synuclein-induced tubule formation in lipid bilayers. The journal of physical chemistry. B, 2011. 115(19): p. 5886-93.

47. Zimmermann, M.T., et al., Free energies for coarse-grained proteins by integrating multibody statistical contact potentials with entropies from elastic network models. Journal of structural and functional genomics, 2011. 12(2): p. 137-47.

48. Lovas, S.Z., Y.; Yu,J.; Lyubchenko, Y.L. . Insight into A $\beta$ misfolding and aggregation. in 32nd European Peptide Symposium. 2012. Budapest.

49. Lv, Z., et al., Nanoprobing of the Effect of Cu(2+) Cations on Misfolding, Interaction and Aggregation of Amyloid beta Peptide. Journal of neuroimmune pharmacology : the official journal of the Society on NeuroImmune Pharmacology, 2012.

50. Lyubchenko, Y.L., DNA and protein-DNA complexes, in Atomic Force Microscopy in Liquid, R.G.R. A. Baro, Editor 2012, Wiley-VCH Verlag GmbH \& Co. KGaA.

51. Lyubchenko, Y.L., A.V. Krasnoslobodtsev, and S. Luca, Fibrillogenesis of huntingtin and other glutamine containing proteins, in Protein Aggregation and Fibrillogenesis in Cerebral and Systemic Amyloid Disease, J.R. Harris, Editor 2012, Springer Netherlands. p. 225-51.

52. Portillo, A.M., A.V. Krasnoslobodtsev, and Y.L. Lyubchenko, Effect of electrostatics on aggregation of prion protein Sup35 peptide. Journal of physics. Condensed matter : an Institute of Physics journal, 2012. 24(16): p. 164205.

53. Krasnoslobodtsev, A.V., Ivan Volkov, Josephat M. Asiago, Jagadish Hindupur, Jean-Christophe Rochet and Yuri L. Lyubchenko, Folding of alpha-synuclein assessed with AFM force spectroscopy: Effect of pathogenic mutations. submitted, 2013.

54. Krasnoslobodtsev, A.V., Jean-Christophe Rochet and Yuri L. Lyubchenko, Nanoimaging for Nanomedicine, in The Biomedical Engineering Handbook, R.B.a.J.D. Bronzino, Editor 2013, CRC Press/Taylor Francis.

55. Lyubchenko, Y.L., Nanoimaging of aggregated proteins: New looks of old folks in Bio-nanoimaging in protein misfolding and aggregation, v.U. Yuri Lyubchenko, Editor 2013.

56. Lyubchenko, Y.L., AFM visualization of protein-DNA interactions, in Single-molecule Studies of Proteins. Series: Biophysics for the Life Sciences., A. Oberhauser, Editor 2013, Springer Science.

57. Lyubchenko, Y.L., Alexey V. Krasnoslobodtsev and Sorin Luca Fibrillogenesis of Huntingtin and Other Glutamine Containing Proteins, in Protein Aggregation and Fibrillogenesis in Cerebral and Systemic Amyloid Disease, R. Harris, Editor 2013, Springer.

58. Lyubchenko, Y.L. and A.A.G.a.L.S. Shlyakhtenko, Visualization of DNA and Protein-DNA Complexes with Atomic Force Microscopy, in Electron Microscopy: Protocols, Third Edition, J. Kuo, Editor 2013, Humana Press.

59. Lovas, S., Yuliang Zhang, Junping Yu, and Yuri L. Lyubchenko, Molecular Mechanism of Ab(13-23) misfolding and aggregation. J. Phys. Chem. , 2013. submitted.

60. Shlyakhtenko, L.S., A. A. Gall and Yuri L Lyubchenko Functionalization for Imaging of DNA and Protein-DNA Complexes with Atomic Force Microscopy, in Methods in molecular biology. Cell Imaging techniques, D.J.T.a.J. Roth, Editor 2013, Humana Press: New York, Heidelberg, London. p. 295-312.

61. Shlyakhtenko, L.S., A.A. Gall, and Y.L. Lyubchenko, Mica functionalization for imaging of DNA and protein-DNA complexes with atomic force microscopy, in Cell Imaging Techniques. Methods and Protocols, J.R. Douglas J. Taatjes, Editor 2013, Humana Press. p. 295-312. 


\section{Outcome of the award DE-FG02-08ER64579}

Under the support from this award we published 34 papers including 24 publications in referred journals, 8 book chapters and one conference proceedings. The list of publication is attached at the end of this report. A few more papers are in preparation. The results of the research were presented at numerous national and international meetings with more than 50 oral and poster presentations.

This award helps us to generate additional funding for federal and local agencies. The list of current, pending and recent grants is given below.

\section{CURRENT SUPPORT}

1 P01 GM091743-01A1 (Harris) 01/01/2011 - 12/31/2016 2.40 calendar NIHINIGMS \$1,332,170 (Lyubchenko budget)

Critical Interactions of APOBEC3's: Molecular Approaches to Novel HIV Therapies

This Program Project (P01) grant application submitted by the University of

Minnesota (Reuben Harris, PI) proposes to combine expertise in molecular virology (Project 1), NMR spectroscopy (Project 2), X-ray crystallography (Project 3), AFM force spectroscopy (Project 4, Lyubchenko) and biochemistry (Project 5) to define APOBEC3 (A3) and HIV-1 Vif protein interactions.

EPS - 1004094 (Choobineh) 10/1/10 - 9/30/15 0.76 calendar

NSF \$398,679 (Lyubchenko budget)

RII: Nanohybrid Functional Materials

This project invests in two independent research areas of local and national importance by capitalizing on Nebraska's core strengths in nanomaterials and algal biology. It will establish two interdisciplinary research centers of excellence: the Center for

Nanohybrid Functional Materials (Nanohybrid Center) and the Nebraska Center for Algal Biology and Biotechnology (Algal Center). These centers will assemble the expertise of 29 faculty members from seven disciplines to collaboratively address important fundamental science questions that also align with state and NSF priorities in nanoscience and energy. Project of Y. Lyubchenko is to study nanomaterials with the use of AFM.

1 R01 GM096039-01A1 (Lyubchenko) 08/01/2011 - 07/31/2015 3.0 calendar NIH/NIGMS \$1,128,600

Protein Self-Assembly into Nanoaggregates

The long-term goal is to understand the mechanisms by which a protein misfolds and self assembles into nanoaggrates. Three major aims are proposed. Identify key nuclei for the aggregation kinetics. Image directly early stages of the self-assembly process. Develop novel linkers and tethers for AFM experiments.

NEBRASKA RESEARCH INITATIVE-NRI 07/01/2011 - 06/30/2012

AFM/Raman Instrument for Nanoimaging Core Facility $\$ 631,668$

Integrated Raman Spectroscopy-Atomic Force Microscope Instrument (AFM/Raman) 


\section{PENDING GRANTS}

1 P41 GM104602-01A1 (Lyubchenko) 7/1/2013 - 6/30/2018

NIH/NIGMS $\$ 5,034,770$ (total cost)

Nanoimaging Center for Biomedicine

1R01GM100156-01A1 (MPI: Bianco, Lyubchenko) 12/01/2012 - 11/30/2017

NIH/NIGMS \$608,918 ((total cost for Lyubchenko)

Mechanistic studies of stalled DNA replication fork rescue

1R01GM107304-01 (Buck, Lyubchenko) 07/01/2013 - 06/30/2017

NIH/NIAID \$469,322 (total cost Lyubchenko)

Mechanistic characterization of transcription factor binding within chromatin

\section{RECENT FEDERAL AND STATE GRANTS}

7/1/07-6/30/11 Lyubchenko \$494,777

NRI (Nebraska Research Initiative)

Nanoimaging Core Facility for Molecular Biology, Genetics and Biotechnology

8/15/08 - 8/14/10 EPS-0701892 (Choobineh) \$387,772 (Lyubchenko)

NSF RII: Nano-enhanced epigenetics research (NE2R)

9/1/06 - 8/31/10 PHY-0615590 (Lyubchenko) \$434,217

NSF

Single Molecule Studies of Recombinational DNA Structure and Dynamics 


\section{List of publications supported by DE-FG02-08ER64579 award}

1. Yu, J., S. Malkova, and Y.L. Lyubchenko, alpha-Synuclein misfolding: single molecule AFM force spectroscopy study. J Mol Biol, 2008. 384(4): p. 992-1001.

2. Lyubchenko, Y.L. and L.S. Shlyakhtenko, AFM for analysis of structure and dynamics of DNA and protein-DNA complexes. Methods, 2009. 47(3): p. 206-13.

3. Lyubchenko, Y.L., L.S. Shlyakhtenko, and A.A. Gall, Atomic force microscopy imaging and probing of DNA, proteins, and protein DNA complexes: silatrane surface chemistry. Methods Mol Biol, 2009. 543: p. 337-51.

4. Rubinstein, A., Y.L. Lyubchenko, and S. Sherman, Dynamic properties of $\mathrm{pH}$ dependent structural organization of the amyloidogenic beta-protein (1-40). Prion, 2009. 3(1): p. 31-43.

5. Yu, J. and Y.L. Lyubchenko, Early stages for Parkinson's development: alpha-synuclein misfolding and aggregation. J Neuroimmune Pharmacol, 2009. 4(1): p. 10-6.

6. Feng, Y., A. Kloczkowski, and R.L. Jernigan, Potentials ' $R$ ' Us web-server for protein energy estimations with coarse-grained knowledge-based potentials. BMC bioinformatics, 2010. 11: p. 92.

7. Haque, F., et al., Adsorption of alpha-synuclein on lipid bilayers: modulating the structure and stability of protein assemblies. The journal of physical chemistry. B, 2010. 114(11): p. 4070-81.

8. Krasnoslobodtsev, A.V., et al., Nanoimaging for prion related diseases. Prion, 2010. 4(4): p. 265-74.

9. Lyubchenko, Y.L., et al., Nanoimaging for protein misfolding diseases. Wiley Interdiscip Rev Nanomed Nanobiotechnol, 2010. 2(5): p. 526-43.

10. Rashin, A.A., A.H. Rashin, and R.L. Jernigan, Diversity of function-related conformational changes in proteins: coordinate uncertainty, fragment rigidity, and stability. Biochemistry, 2010. 49(27): p. 5683-704.

11. Kim, B.H., et al., Single-Molecule Atomic Force Microscopy Force Spectroscopy Study of Abeta-40 Interactions. Biochemistry, 2011. 50(23): p. 5154-5162.

12. Krasnoslobodtsev, A.V., B.-H. Kim, and Y.L. Lyubchenko, Nanoimaging for protein misfolding diseases. Critical role of misfolded dimers in the amyloid self-assembly. Microsc. Microanal. 17 (Suppl 2), 2011.17(Suppl 2): p. 170-171.

13. Lyubchenko, Y.L., Preparation of DNA and nucleoprotein samples for AFM imaging. Micron, 2011. 42(2): p. 196-206.

14. Lyubchenko, Y.L., AFM imaging in liquid of DNA and protein-DNA complexes, in AFM in Liquids, A.M.a.R. Baro, R. , Editor 2011, Wiley-VCH.

15. Lyubchenko, Y.L., L.S. Shlyakhtenko, and T. Ando, Imaging of nucleic acids with atomic force microscopy. Methods, 2011. 54(2): p. 274-83.

16. Pandey, A.P., et al., alpha-Synuclein-induced tubule formation in lipid bilayers. The journal of physical chemistry. B, 2011. 115(19): p. 5886-93.

17. Yu, J., J. Warnke, and Y.L. Lyubchenko, Nanoprobing of alpha-synuclein misfolding and aggregation with atomic force microscopy. Nanomedicine : nanotechnology, biology, and medicine, 2011. 7(2): p. 146-52. 
18. Zimmermann, M.T., et al., Free energies for coarse-grained proteins by integrating multibody statistical contact potentials with entropies from elastic network models. Journal of structural and functional genomics, 2011. 12(2): p. 137-47.

19. Krasnoslobodtsev, A.V., et al., Effect of spermidine on misfolding and interactions of alpha-synuclein. PLoS ONE, 2012. 7(5): p. e38099.

20. Lovas, S.Z., Y.; Yu,J.; Lyubchenko, Y.L. . Insight into A $\beta$ misfolding and aggregation. in 32nd European Peptide Symposium. 2012. Budapest.

21. Lv, Z., et al., Nanoprobing of the Effect of Cu(2+) Cations on Misfolding, Interaction and Aggregation of Amyloid beta Peptide. Journal of neuroimmune pharmacology : the official journal of the Society on NeuroImmune Pharmacology, 2012.

22. Lyubchenko, Y.L., DNA and protein-DNA complexes, in Atomic Force Microscopy in Liquid, R.G.R. A. Baro, Editor 2012, Wiley-VCH Verlag GmbH \& Co. KGaA.

23. Lyubchenko, Y.L., A.V. Krasnoslobodtsev, and S. Luca, Fibrillogenesis of huntingtin and other glutamine containing proteins, in Protein Aggregation and Fibrillogenesis in Cerebral and Systemic Amyloid Disease, J.R. Harris, Editor 2012, Springer Netherlands. p. 225-51.

24. Portillo, A.M., A.V. Krasnoslobodtsev, and Y.L. Lyubchenko, Effect of electrostatics on aggregation of prion protein Sup35 peptide. Journal of physics. Condensed matter : an Institute of Physics journal, 2012. 24(16): p. 164205.

25. Krasnoslobodtsev, A.V., Ivan Volkov, Josephat M. Asiago, Jagadish Hindupur, JeanChristophe Rochet and Yuri L. Lyubchenko, Folding of alpha-synuclein assessed with AFM force spectroscopy: Effect of pathogenic mutations. submitted, 2013.

26. Krasnoslobodtsev, A.V., Jean-Christophe Rochet and Yuri L. Lyubchenko, Nanoimaging for Nanomedicine, in The Biomedical Engineering Handbook, R.B.a.J.D. Bronzino, Editor 2013, CRC Press/Taylor Francis.

27. Lyubchenko, Y.L., Nanoimaging of aggregated proteins: New looks of old folks in Bionanoimaging in protein misfolding and aggregation, v.U. Yuri Lyubchenko, Editor 2013.

28. Lyubchenko, Y.L., AFM visualization of protein-DNA interactions, in Single-molecule Studies of Proteins. Series: Biophysics for the Life Sciences., A. Oberhauser, Editor 2013, Springer Science.

29. Lyubchenko, Y.L., Alexey V. Krasnoslobodtsev and Sorin Luca Fibrillogenesis of Huntingtin and Other Glutamine Containing Proteins, in Protein Aggregation and Fibrillogenesis in Cerebral and Systemic Amyloid Disease, R. Harris, Editor 2013, Springer.

30. Lyubchenko, Y.L. and A.A. Gall and L.S. Shlyakhtenko, Visualization of DNA and Protein-DNA Complexes with Atomic Force Microscopy, in Electron Microscopy: Protocols, Third Edition, J. Kuo, Editor 2013, Humana Press.

31. Lovas, S., Yuliang Zhang, Junping Yu, and Yuri L. Lyubchenko, Molecular Mechanism of Ab(13-23) misfolding and aggregation. J. Phys. Chem. , 2013. submitted.

32. Shlyakhtenko, L.S., A. A. Gall and Yuri L Lyubchenko Functionalization for Imaging of DNA and Protein-DNA Complexes with Atomic Force Microscopy, in Methods in molecular biology. Cell Imaging techniques, D.J.T.a.J. Roth, Editor 2013, Humana Press: New York, Heidelberg, London. p. 295-312.

33. Shlyakhtenko, L.S., A.A. Gall, and Y.L. Lyubchenko, Mica functionalization for imaging of DNA and protein-DNA complexes with atomic force microscopy, in Cell Imaging 
Techniques. Methods and Protocols, J.R. Douglas J. Taatjes, Editor 2013, Humana Press. p. 295-312.

34. Tong, Z., Andrew Mikheikin, Alexey Krasnoslobodtsev, Zhengjian Lv and Yuri L. Lyubchenko, Novel Polymer Linkers for Single Molecule AFM Force Spectroscopy. Methods, 2013. in press. 